\title{
Novel technique to manage pacemaker exposure with buried flap reconstruction: case series
}

\author{
Raja Tiwari, ${ }^{1}$ Shruti Marwah, ${ }^{1}$ Ambuj Roy, ${ }^{2}$ Maneesh Singhal ${ }^{1}$
}

- Additional material is

published online only. To view please visit the journal online (http://dx.doi.org/10.1136/ heartasia-2018-011086).

${ }^{1}$ Department of Plastic Surgery, All India Institute of Medical Sciences, New Delhi, India ${ }^{2}$ Department of Cardiology, All India Institute of Medical Sciences, New Delhi, India

\section{Correspondence to}

Dr Raja Tiwari, Department of Plastic surgery, All India Institute of Medical Sciences, New Delhi 110019, India; tiwariraja1981@ gmail.com

Received 26 August 2018 Revised 14 November 2018 Accepted 16 November 2018

Check for updates

(C) Author(s) (or their employer(s)) 2019. No commercial re-use. See rights and permissions. Published by BMJ.

To cite: Tiwari $R$, Marwah $S$, Roy $\mathrm{A}$, et al. Heart Asia

2019:11:e011086

doi:10.1136/

heartasia-2018-011086

\section{ABSTRACT}

Objective Exposure of implantable electrical devices may increase morbidity and mortality significantly. Usually superficial infections are conservatively managed whereas invasive infections necessitate complete capsulectomy, subpectoral placement or implant exchange. Most commonly inadequate soft tissue coverage, soft tissue thinning and scar dehiscence over the edge of the pacemaker are the primary predisposing event. Multiple local surgical options have been described, however, with all these designs, the final scar still remains over the edge of the pacemaker and continue to have a tendency of thinning out with time. We have described a local skin flap which can be deepithelialized and partially buried under the skin to increase the thickness over the pacemaker edge, thereby preventing further recurrence.

Methods Three patients admitted in the Cardiology Department presented with impending exposure $(n=2)$ and exposed implant $(n=1)$ over the edge of pacemaker with superficial infection. Local modified rotation skin flap which was de-epithelialized and partially buried under the skin to increase the thickness over the pacemaker edge was performed under local anaesthesia in all the cases.

Results Flaps settled well in all patients with no evidence of infection, scar dehiscence and recurrence over a followup period of 2 years.

Conclusions This flap technique is recommended for cases of impending pacemaker exposure resulting due to scar dehiscence over the edge and helps by addressing the predisposing factors at an initial stage. In our experience, this technique also helped to salvage exposed pacemaker with superficial infection. To our bestof knowledge this technique has not been described before in the literature.

Exposure of implantable electrical devices though uncommon $^{1}$ results in bacterial contamination and subsequent infection, which may increase mortality significantly. ${ }^{2}$ Secondary operative procedures may be required, and these may increase morbidity in such patients. Most commonly, inadequate soft tissue coverage in thin-built patients, soft tissue thinning over time and scar dehiscence over the edge of the pacemaker are considered the primary predisposing events. This thinned scar is prone to both infection and to mechanical irritation from chemical or immunological irritants. Several risk factors have been studied, including administration of anticoagulants or antiplatelet regimen, presence of cutaneous lesions or other signs and symptoms of infection, or pocket haematoma, ${ }^{3}$ which may predispose to subsequent implant exposure. In addition, continuous pressure from an automobile seat belt, bra strap or even purse shoulder strap may sometimes cause pain and tenderness over the thinned edge of the pacemaker. ${ }^{4}$

\section{Key messages}

What is already known about this subject?

- Exposure of implantable electrical devices is an uncommon occurrence, but when it occurs it may result in bacterial contamination and subsequent infection, leading to life-threatening complications.

- Several methods of management have been described, ranging from conservative to extensive surgical procedures; however, most of them are extensive and associated with morbidity.

What does this study add?

- We aim to describe a short procedure that can be performed in patients with exposed or impending exposure of pacemaker devices, under local anaesthesia in a day care setting, with minimum morbidity.

- It is a novel technique that has not been described before and we believe it can be easily performed by cardiologists.

How might this impact on clinical practice?

- Pacemaker device once it gets infected may need to be replaced completely, and this adds to patient morbidity.

- However if we intervene at an early stage and prevent the pacemaker from getting infected, it might save us from the antecedent morbidity and cost.

Numerous lines of management of exposed pacemaker devices have been described. Conservative management with antibiotics, irrigation with n-acetylcysteine solution, complete capsulectomy, dermal grafts, ${ }^{4}$ subpectoral placement, implantation at the contralateral side or muscle flap coverage ${ }^{5}$ are few to name. Retained infectious devices, however, continue to remain a source of relapse, which is closely related to a higher mortality rate. ${ }^{6}$ The risk of infection is substantially less if the infection is superficial and implant and leads are impending exposure but not yet been exposed to contaminants. In such cases of impending exposure, local flaps can provide a good option to prevent invasive infection and salvage the implant.

Local flap options include random pattern flaps ${ }^{7}$ and pectoral perforator-based propeller flaps. ${ }^{5}$ However, with all these designs, the final scar still remains over the edge of the pacemaker and continues to have a tendency of thinning out with time and thus a potential risk of re-exposure in the future.

In order to obtain thicker skin coverage especially over the edge of the pacemaker, we suggest use of 
a modified rotation flap with excised Burow's triangles and with an advancing edge of buried de-epithelialised soft tissue. This advancing edge of de-epithelialised skin and subcutaneous tissue is folded on itself and then buried under the skin overlying the pacemaker edge. This is expected to reinforce the thickness of the skin at the edge and reduce the risk of a re-exposure and associated complications. $^{5}$

This technique was carried out in three patients to evaluate the efficacy and results of our buried modified rotation flap for coverage of impending pacemaker exposure.

\section{METHODOLOGY}

Three patients admitted in the cardiology department of All India Institute of Medical Sciences, New Delhi, for treatment of various conduction blocks were chosen for the study. All these patients had impending pacemaker exposure or scar dehiscence over the edge of the pacemaker within 3 months of implant placement. A thorough history was taken and physical examination was done to rule out any other comorbid illness. Patients were assessed for feasibility of the procedure under local anaesthesia, including status of cooperation and pain sensitivity. Detailed consent was taken after explaining the nature of the procedure, and it was explained that subsequent procedures may be needed in case there was recurrence with the current procedure. A routine laboratory work-up was done to assess haemoglobin, protein levels and blood sugar levels, and to ensure adequate kidney function. All patients were then scheduled for surgery.

\section{Preoperative protocol}

In all patients we followed the preoperative protocol of changing the pacemaker mode to asynchronous in order to avoid interference while using cautery.

\section{Operative procedure}

The surgical procedure was conducted in supine position with unilateral chest in the operative field. The procedure was performed under local anaesthesia, and 2\% lignocaine with epinephrine (1:1 dilution with normal saline) was infiltrated in all patients, with the total dose limit depending on the weight. All patients were given $1 \mathrm{~g}$ injection of amoxicillin/clavulanic acid just before the placement of incision. We recommend using magnifying loupes $(2.5 \times)$ as they increase precision with surgery, and it becomes easier to identify any tears in the implant capsule if they happen. A triangular segment of the skin was excised, including the thinned scar or sinus. In one case the pacemaker capsule was torn exposing the pacemaker, so a culture was taken from the capsule of the pacemaker.

A modified rotation flap was marked after taking into account the amount of skin laxity present in the infraclavicular region (refer to figure 1). In two cases the base of the flap was lateral and it was medially based in one case. De-epithelialisation was done over the advancing edge of the rotating flap. The skin margin over the pacemaker's edge was undermined, and the rotation flap was buried under the undermined skin and anchored to a capsule using 4-0 polydioxanone sutures, thereby overlapping and increasing the thickness of the skin at the pacemaker edge. The rest of the flap was sutured in two layers using 4-0 polydioxanone for subdermal stitching and 4-0 Prolene suture for skin stitches. The dog ear thus created because of flap advancement was removed by excising a small Burow's triangle. ${ }^{8}$ This excision of Burow's triangle also distributed and decreased the tension at the final suture line.

The optimum size of the de-epithelialised segment was twice the width of the excised scar. The size of the rotation flap was 1.5-2.5 times the size of the de-epithelialised segment. The size of the

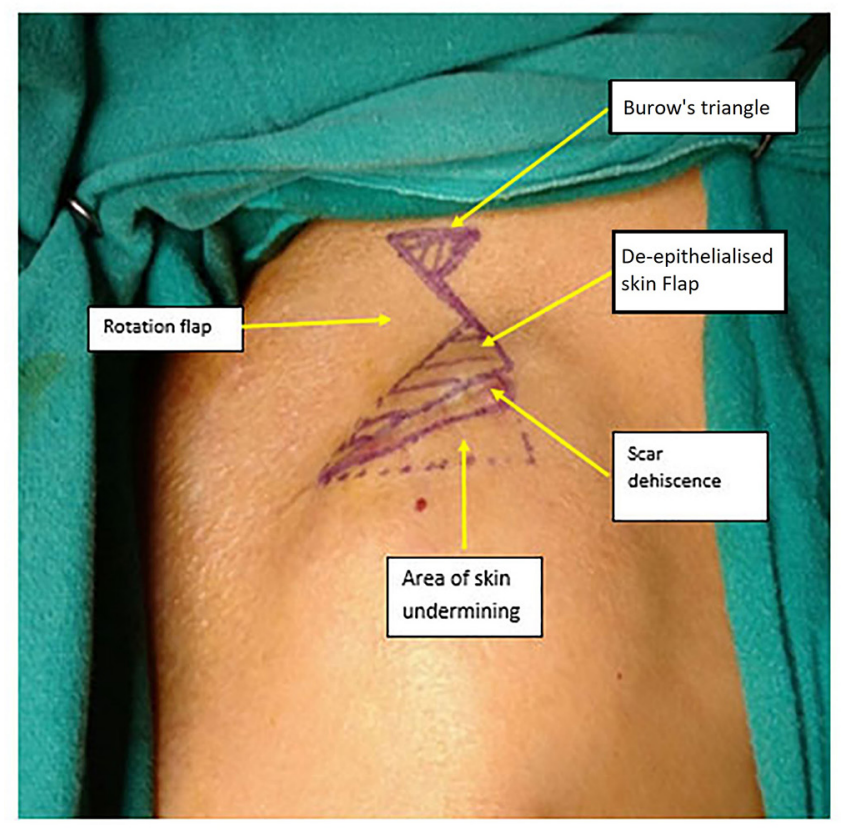

Figure 1 Design of the modified rotation flap is marked after taking into account the amount of skin laxity present in the infraclavicular region. The shaded area is the de-epithelialised skin which is buried.

Burow's triangle was dependent on the amount of Burow's triangle thus created. The flap contained skin, adipose tissue with its underlying fascia not including the muscle, hence can also be termed as fascio-adipo-cutaneous flap. The flap raised was of uniform thickness until its base. Haemostasis was ensured before skin closure. Drains were not required; however, compression over the dressing was provided with elastic tapes.

\section{Postoperative protocol}

The patients were monitored for evidence of any conduction abnormality or pacemaker malfunction on the day of operation. Injectable antibiotic (amoxicillin/clavulanic acid) was continued for 24 hours. Patients were discharged the next day on oral antibiotics, which were continued for 6 days. All patients were followed in the plastic surgery outpatient clinic for alternate-day dressings to look for any collection of blood or serum. Suture removal was usually done on the tenth postoperative day and then sterile tapes were applied. Silicon gel massage was started at 3 weeks to achieve a well-healed and well-settled scar and was continued for 3 months. Strict sugar and blood pressure control was followed to achieve better and quicker results.

\section{RESULTS}

The details of each patient, including the duration of surgery and follow-up period, are mentioned below. All patients recovered well and were discharged within 2-3 days of admission. None of the three patients had any complications. None of them had any evidence of subsequent implant exposure over the period of follow-up.

\section{Case 1}

A 40-year-old man was referred to us with complaint of impending pacemaker exposure 2 months after placement. A laterally based flap was done with de-epithelialised part buried under the edge of the scar. The total operative time was $30 \mathrm{~min}$. Uneventful recovery took place with no evidence of recurrence even after 25 months of follow-up (refer to figure 2). 


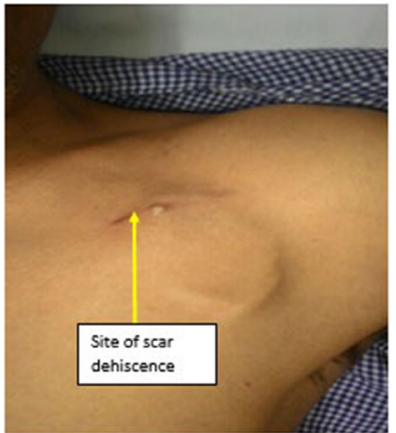

Scar dehiscence with impending exposure

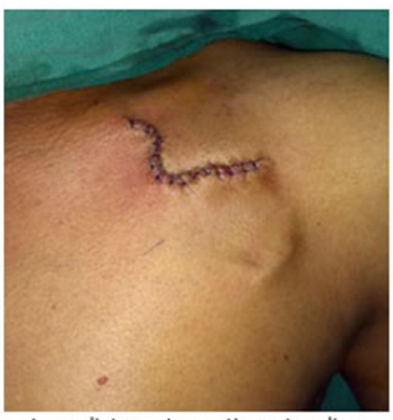

Immediate postoperative suture line

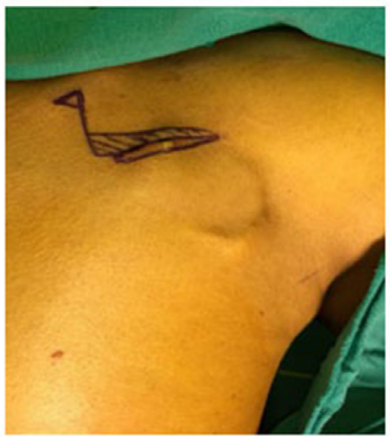

Flap and scar excision markings

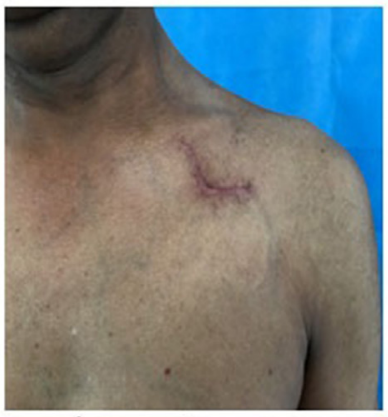

Follow-up after one year
Figure 2 A laterally based modified rotation flap was done with deepithelialised part buried under the edge of the scar.

\section{Case 2}

A 60-year-old man was referred to us with complaint of impending pacemaker exposure within a month after placement. A medially based flap was done with de-epithelialised part buried under the edge of the scar. The total operative time was $30 \mathrm{~min}$. Uneventful

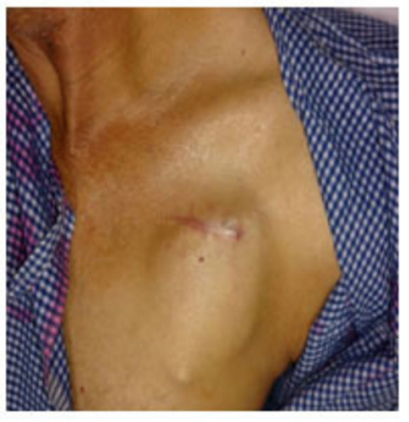

Scar thinning over the edge

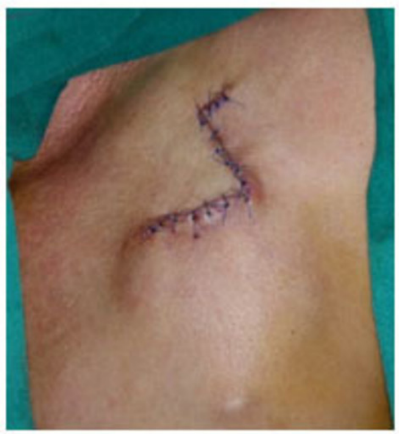

Immediate Postoperative suture line

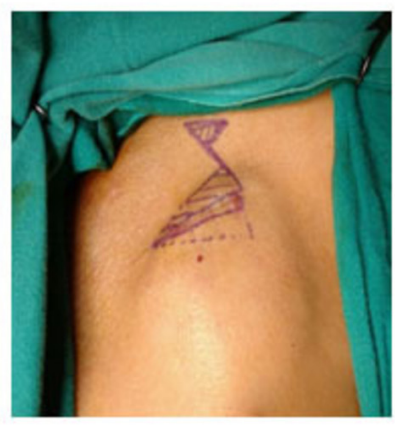

Flap and scar excision markings

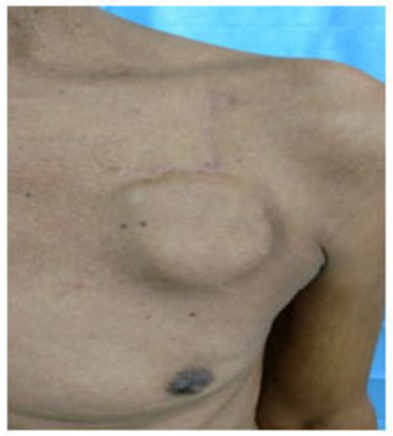

Follow-up at one year
Figure 3 A medially modified rotation based flap was done with deepithelialised part buried under the edge of the scar.

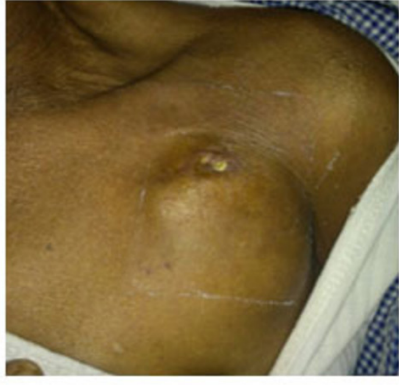

Scar dehiscence with exposed implant

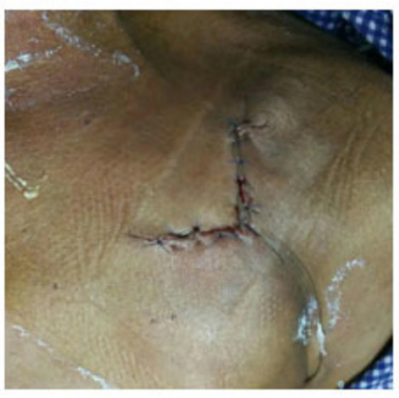

Immediate postoperative suture line

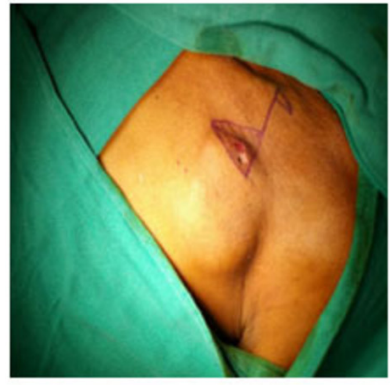

Flap and scar excision markings

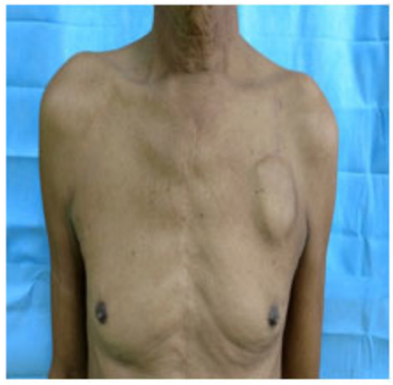

Follow-up at one year
Figure 4 A case of exposed implant but sterile on culture. A medially modified rotation based flap was done with de-epithelialised part buried under the edge of the scar.

recovery took place with no evidence of recurrence after 28 months of follow-up (refer to figure 3).

\section{Case 3}

A 45-year-old patient had presented to us after 2.5 months of pacemaker placement and scar dehiscence with pacemaker exposure for 2 days. Pus cultures taken from the exposed part were sterile. Hence, decision was taken to cover the exposed part with local flap after thorough cleaning of the pacemaker with antibiotic solution (refer to figure 4). The total operative time was $45 \mathrm{~min}$. No complications were observed postoperatively and the implant was retained at 20 months' follow-up.

\section{DISCUSSION}

Implanted permanent pacemakers cause erosion or infection in around $0.13 \%-12.6 \%$ of patients. ${ }^{9}$ Limited literature is available on exposure management, with various recommendations such as removal and replacement in a secondary operation several months later often being advocated for pacemakers.

Several measures have been advocated to salvage exposed devices and minimise risks of infection or re-exposure. Complete capsulectomy and accurate cleaning of the pocket have been recommended. Routine irrigation of the pocket with an n-acetylcysteine solution, being a glutathione precursor, has shown to be effective in destroying bacterial biofilms and inhibiting their formation. ${ }^{10}$ For clean-contaminated surgery, a single dose of preoperative prophylactic antibiotic can be administered. In a series of 17 patients with exposed venous and electrical devices, described by Toia et $a l^{5}$ after irrigation and without postoperative antibiotics, no infection was observed. ${ }^{5}$

Several authors recommend pocket change and subpectoral placement, but there is no general agreement and different techniques have been suggested. ${ }^{11-13}$ Fayman et al ${ }^{12}$ reported subpectoral placement for 13 patients of impending cardiac pacemaker with a success rate of $84.6 \%$. Not only the subpectoral placement 
is a major operation requiring general anaesthesia but also has a perioperative complication rate of $7.7 \%$ and a late extrusion rate of $7.7 \% .^{12}$

Bonawitz ${ }^{14}$ in a series of 16 patients with exposed pacemakers reported no difference in re-exposure rate for submuscular or subfascial placement, the latter being the preferred choice. For pacemakers or other voluminous electrical devices, submuscular placement is preferred because exposure in the absence of infection is often due to an imbalance between skin thickness and pressure exerted by the device; therefore, if the primary cause (mechanical pressure) of exposure is not eliminated, re-exposure may happen.

Also, submuscular placement of the pacemaker allows for a better aesthetic outcome and has been proposed as first choice even for primary pacemaker placement. ${ }^{15}$ However, subpectoral placement has a longer learning curve especially for the cardiologists who primarily place these devices and may be associated with discomfort and spasm in some cases, as reported by Bonawitz ${ }^{14}$ and Fayman et al. ${ }^{12}$

A surgical technique involving wide excision of the exposure site with a rhombic incision pattern, followed by removal of the generator unit, complete debridement of the peridevice capsule, implantation of a new device and transposition of a rhombic flap, has also been described by Kolker et al. ${ }^{7}$ Again this is an extensive technique, requires general anaesthesia for change of device and the final suture line still remains over the edge of the pacemaker. Thus the chances of re-exposure are not diminished.

A series demonstrating the salvage of cardiac devices with anterior muscle-splitting and subpectoral repositioning as a technically feasible approach with favourable outcomes and low morbidity has also been described, ${ }^{16}$ with limitations in the form of technical difficulties and anaesthesia requirements.

Our flap design is based on the principles advocated by Karydakis in $1992 .{ }^{17} \mathrm{He}$ had opined that in pilonidal sinus, by shifting the gluteal cleft to the lateral gluteal region using a local advancement flap, the vulnerability of the skin to the insertion of hair at the depth of the natal cleft decreases. Similarly we tried to eliminate the primary causative influence, that is, thinning of the scar at the pacemaker's edge, by increasing the thickness of skin by placing a folded de-epithelialised skin flap under the skin. Excision of Burow's triangles in addition help to decrease the tension on the final suture line and improve the skin contours.

Even though several kinds of local flaps have been recommended, this particular flap has several advantages. It can be performed under local anaesthesia, thereby minimising the risks of general anaesthesia especially in patients with cardiac disease. Second minimal dissection and handling is required as compared with other techniques such as subpectoral placement. This significantly limits the complication rate due to surgery and due to displacement of wires. Lastly increased thickness at the edge of the pacemaker prevents any further recurrence of the problem. This cannot be achieved by any other local flaps commonly described in the literature.

The limiting factors for this procedure may include scarring of the surrounding skin due to previous operations or injury interfering with the elasticity of the skin. Similarly any local infection such as a boil or carbuncle in the area of usable skin is an absolute contraindication for this procedure. Also extreme care has to be taken with haemostasis in patients on anticoagulants and antiplatelet drugs. These if permitted may need to be stopped in advance as part of preoperative preparation. Complications that may occur are haematoma or seroma formation and skin dehiscence, and can be addressed by drainage, lavage and resuturing. Compression over the dressing usually averts these problems.

\section{CONCLUSION}

We recommend modified rotation flap with Burow's triangles as a novel approach for coverage of impending pacemaker exposure. It is also advisable to intervene at an early stage when the scar is developing any signs of thinning rather than when it is already exposed. Impending exposure is easy to manage and with least morbidity to the patient. As per our experience, this technique also worked in case of an exposed implant where the culture was sterile. However a larger series is needed to recommend this technique for all cases of exposed pacemaker devices. We also would like to advise that the pacemaker device should be inserted such that the edge is at least $2 \mathrm{~cm}$ from the suture line to prevent dehiscence, which is the predisposing factor in most cases.

Contributors RT: Substantial contributions to the conception or design of the work along with performing the operations and the acquisition, analysis, or interpretation of data for the work. MS: critical revision and final approval of the version to be published. SM: acquisition of data, interpretation and drafting the initial work. AR: substantial contributions to the acquisition, analysis and interpretation of data for the work. All authors have read the contributor ship statement and author list. The corresponding author is responsible for signing agreement on their behalf.

Funding The authors have not declared a specific grant for this research from any funding agency in the public, commercial or not-for-profit sectors.

Competing interests None declared.

Patient consent for publication Not required.

Ethics approval Institute Ethics Committee for Post Graduate Research, All India Institute of Medical Sciences, New Delhi.

Provenance and peer review Not commissioned; externally peer reviewed.

\section{REFERENCES}

1 Nery PB, Fernandes R, Nair GM, et al. Device-related infection among patients with pacemakers and implantable defibrillators: incidence, risk factors, and consequences. J Cardiovasc Electrophysiol 2010;21:786-90.

2 Le KY, Sohail MR, Friedman PA, et al. Impact of timing of device removal on mortality in patients with cardiovascular implantable electronic device infections. Heart Rhythm 2011;8:1678-85

3 Klug D, Balde M, Pavin D, et al. Risk factors related to infections of implanted pacemakers and cardioverter-defibrillators: results of a large prospective study. Circulation 2007;116:1349-55.

4 Rudolph R, Smith MR, Curtis GP. Salvage of pacemakers and automatic implantable cardioverter-defibrillators using dermis grafts. Ann Thorac Surg 2011;91:452-6.

5 Toia F, D'Arpa S, Cordova A, et al. Exposed subcutaneous implantable devices: an operative protocol for management and salvage. Plast Reconstr Surg Glob Open 2015:3:e343.

6 Sekiguchi Y. Conservative therapy for the management of cardiac implantable electronic device infection. J Arrhythm 2016;32:293-6.

7 Kolker AR, Redstone JS, Tutela JP. Salvage of exposed implantable cardiac electrical devices and lead systems with pocket change and local flap coverage. Ann Plast Surg 2007;59:26-30.

8 Grassetti L, Lazzeri D, Torresetti M, et al. Aesthetic refinement of the dog ear correction: the $90^{\circ}$ incision technique and review of the literature. Arch Plast Surg 2013;40:268-9.

9 Wade JS, Cobbs CG. Infections in cardiac pacemakers. Curr Clin Top Infect Dis 1988:9:44-61.

10 Zhao T, Liu Y. N-acetylcysteine inhibit biofilms produced by Pseudomonas aeruginosa. BMC Microbiol 2010;10:140.

11 Griffith MJ, Mounsey JP, Bexton RS, et al. Mechanical, but not infective, pacemaker erosion may be successfully managed by re-implantation of pacemakers. $\mathrm{Br}$ Heart J 1994;71:202-5.

12 Fayman MS, Chait LA, Orak F. A subpectoral pocket in the management of a patient with impending extrusion of a pulse generator. Plast Reconstr Surg 1986;78:182-5.

13 Garcia-Rinaldi R, Revuelta JM, Bonnington L, et al. The exposed cardiac pacemaker. Treatment by subfascial pocket relocation. J Thorac Cardiovasc Surg 1985;89:136-41.

14 Bonawitz SC. Management of exposure of cardiac pacemaker systems. Ann Plast Surg 2012;69:292-5.

15 Persichetti P, Brunetti B, Cagli B, et al. Aesthetic subpectoral placement of implantable cardioverter defibrillators. Ann Plast Surg 2014;72:188-92.

16 Knepp EK, Chopra K, Zahiri HR, et al. An effective technique for salvage of cardiac-related devices. Eplasty 2012;12:e8.

17 Karydakis GE. Easy and successful treatment of pilonidal sinus after explanation of its causative process. Aust N Z J Surg 1992:62:385-9. 